\title{
Análisis del sistema familiar representado en la canción EI gran varón. La canción como recurso didáctico a nivel superior
}

\begin{abstract}
Analysis of the family system depicted in the song El gran varón. Song as an educational resource in higher education
\end{abstract}

Análise do sistema familiar representado na música The Great Man. A música como um recurso de ensino em um nível superior

Eva Yuriria Tapia San Pablo

Universidad Nacional Autónoma de México, Facultad de Estudios Superiores Iztacala

México

eyurita.san@gmail.com https://orcid.org/0000-0001-8953-7573

Iris Xóchitl Galicia Moyeda

Universidad Nacional Autónoma de México, Facultad de Estudios Superiores Iztacala

México

iris@unam.mx https://orcid.org/0000-0002-8287-710X

Jorge Víquez Rodríguez

Universidad Nacional Autónoma de México, Facultad de Estudios Superiores Iztacala

México

jorge.vique@gmail.com https://orcid.org/0000-0001-6849-3508

\section{(c) 9}




\section{Revista Iberoamericana \\ de las Ciencias Sociales y \\ Humanísticas}

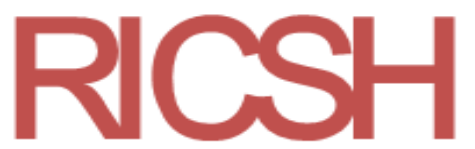

ISSN: $2395-7972$

\section{Resumen}

Las canciones constituyen una importante fuente de difusión de las ideas y de los elementos sociales de mayor representatividad, dado que su desarrollo se entrelaza a las condiciones económicas, sociales e históricas de cada cultura. En tal sentido, el objetivo de este trabajo fue identificar cómo a través de las canciones se proyectan las interacciones familiares durante las diferentes fases del ciclo vital de la familia y el ajuste a un modelo sexual desapegado de la norma. Para ello, y desde una perspectiva sistémica, se analizó la canción El gran varón, pues se observó que a través de su narrativa los protagonistas forman parte de una familia con un estilo de crianza autoritario, tradicional y apegado a las premisas de la realidad presentes en una comunidad. En síntesis, se propone que el análisis de las canciones puede ser un elemento didáctico en niveles educativos superiores para fomentar el proceso de enseñanza-aprendizaje de diversas nociones teóricas de orientación sistémica.

Palabras clave: canciones, ciclo vital familiar, construcción social, estilos de crianza, identidad de género, música.

\section{Abstract}

Songs constitute an important source for the dissemination of ideas and social elements of greater representation, since its development is intertwined to each culture's economic, social and historic conditions. The aim of this paper is to identify how familial interactions during the different phases of a family's life cycle, as well as adjustment to a sexual model detached from the norm, are projected through songs. The song "El gran varón" was analyzed from a systemic perspective, noting that, throughout the song's narrative, the protagonists are part of a family with an authoritarian, traditional parenting style, attached to reality assumptions of a community. Song analysis is recommended as a didactic element in the teaching-learning process of several systemic theoretical notions in higher education.

Keywords: songs, family's life cycle, social construction, parenting styles, gender identity, music. 


\section{Revista Iberoamericana \\ de las Ciencias Sociales y \\ Humanísticas}

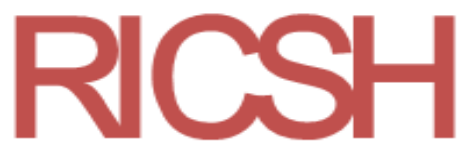

ISSN: $2395-7972$

\section{Resumo}

As músicas constituem uma importante fonte de difusão das idéias e elementos sociais de maior representatividade, uma vez que seu desenvolvimento está entrelaçado com as condições econômicas, sociais e históricas de cada cultura. O objetivo deste trabalho é identificar como, por meio dos cantos, as interações familiares são projetadas durante as diferentes fases do ciclo de vida familiar e o ajustamento de um modelo sexual separado da norma. "El Gran Varón " foi analisado a partir de uma perspectiva sistêmica, observando que, através da narrativa da música, os protagonistas dessa música fazem parte de uma família com um estilo autoritário, tradicional e apegado às premissas da realidade presentes na literatura. uma comunidade Propõe-se que a análise das músicas possa ser um elemento didático nos níveis de ensino superior no processo de ensino-aprendizagem de diversas noções teóricas de orientação sistêmica.

Palavras-chave: músicas, ciclo de vida familiar, construção social, equidade de gênero, estilos parentais.

Fecha Recepción: Mayo 2019

Fecha Aceptación: Diciembre 2019

\section{Introducción}

La música es un elemento productor de colectivos que funge como integrador y transmisor de patrones de comportamiento, ya que su disfrute — además de resultar una experiencia individual - también es una manifestación colectiva (Cuesta y Gómez, 2013). Es decir, la música expresa no solo lo que siente, piensa o realiza el individuo que la compone y/o ejecuta, sino también lo que sienten, piensan o realizan quienes la escuchan. Por ende, una obra musical puede llegar a convertirse en elemento identitario (Hormigos, 2010).

Tomando en consideración que la música es vehículo y expresión de la cultura, resulta importante analizar el papel que desempeña en la difusión de las ideas socialmente aceptadas, en especial en aquellos casos de las canciones más populares, pues son las que mayor impacto tienen en niños, adolescentes y adultos de todos los estratos sociales, económicos y culturales. La canción popular, en efecto, es considerada como una forma importantísima de cultura, de ahí que su análisis sirva como variable para determinar fenómenos identitarios, psicosociales, políticos, antropológicos y estéticos de la sociedad (Muñoz-Hidalgo, 2007). 


\section{Revista Iberoamericana \\ de las Ciencias Sociales y \\ Humanísticas}

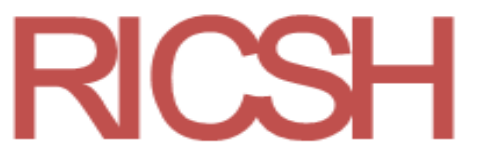

ISSN: 2395 - 7972

Lo anterior pone de manifiesto la importancia de la función comunicativa que radica en el contenido de las canciones, ya que en estas subyacen mensajes e ideas que se difunden entre un público muy amplio. Por eso, resulta significativo mirar con detenimiento las temáticas expuestas en esta forma de manifestación artística, ya que las letras de algunas canciones abordan temas que preocupan a determinados grupos, los cuales se ven reflejados en ellas.

$\mathrm{Al}$ respecto, cabe resaltar que existen diversos estudios que analizan las letras de las canciones desde diferentes enfoques. Por ejemplo, se ha aplicado el análisis crítico del discurso empleando la gramática sistémico-funcional de Halliday a la representación discursiva del espacio urbano en los textos de las canciones del grupo musical francés Zebda. Las canciones examinadas muestran el espacio como elemento socializador, incluso en ámbitos vinculados con la exclusión social. Las canciones dedicadas a las chabolas aluden a franjas de la ciudad forjadas por la inmigración y caracterizadas por el mal diseño urbanístico, del cual los ciudadanos han sido víctimas. Sin embargo, este espacio siempre aparece en las canciones como un punto de encuentro común del que el emisor se siente parte integrante (Iglesias, 2011).

Otras indagaciones profundizan en la violencia de género de las canciones actuales. Gallucci (2008), desde el análisis crítico del discurso, describe y explica la imagen de la mujer que se representa en las letras del reggaeton, para lo cual se toman en consideración los contextos en que se producen los textos y las ideologías manifestadas en el discurso. Este análisis revela que son canciones pobres en contenido y que la orientación de las letras se enfoca de forma predominante en un contenido sexual que denigra a la mujer.

También hay estudios sobre los roles de género en la cultura popular, específicamente en los tangos "clásicos". Al respecto, Juliano (1998) muestra cómo se ve reflejado el discurso del sector masculino de la población, lo que permite entender el cambio de roles de género producido en un sector de la población, donde las mujeres adquirían creciente protagonismo aunque guardaran silencio.

Por otra parte, Berrocal y Gutiérrez (2002) evalúan el contenido en una muestra de canciones populares españolas para conocer el papel del género latente en ellas. En sus resultados, además de reportar la existencia de estereotipos masculinos y femeninos, los autores dan cuenta de un tema poco común en las letras de las canciones: la nula toma de decisión por parte de la mujer en la elección del esposo. 


\section{Revista Iberoamericana \\ de las Ciencias Sociales y \\ Humanísticas}

ISSN: 2395 - 7972

Muñoz-Hidalgo (2007), en cambio, se enfoca en la letra de los boleros con el objeto de precisar si esa escritura se corresponde con un género literario determinado. El autor concluye que el bolero llega a configurar un imaginario amoroso que la poesía modernista no perfila con igual precisión debido a que en sus letras el discurso de amor es redundante, donde se pueden identificar unidades discursivas mayores de fácil señalamiento. En este sentido, el investigador sugiere un evidente paralelismo entre el bolero y la poesía cortesana medieval. Añade que aunque los boleristas, a diferencia de los trovadores medievales, tienen una censura de índole religioso-moral, esta es superada por la evidente complicidad de la industria cultural, que alienta la circulación bolerística por razones comerciales.

Por su parte, De la Peza (2001) estudia las condiciones sociales de emergencia del bolero y analiza la función educativa informal que tuvo este género musical en una época determinada. La autora se centra en la significación del bolero de acuerdo con las diferentes materialidades y lugares que este asigna al sujeto amoroso con el fin de abordar la educación sentimental en diferentes grupos sociales en México.

Muñoz-Hidalgo (2007) también trabaja con el bolero analizando el discurso en piezas clásicas de dicho género. En los resultados se aprecia el imaginario colectivo del amor presente en esas canciones y algunas derivaciones culturales de ese imaginario para la conformación de contenidos identitarios de la cultura latinoamericana. Sobre este aspecto, hay que señalar que para De la Peza el bolero es un género musical que ha ayudado a la educación informal del área sentimental de los individuos de diversas generaciones y nacionalidades, lo que es congruente con la propuesta de Colomo (2014), quien menciona que las canciones, por tratarse de un elemento cultural y por su capacidad de comunicación, se convierten en auténticos vehículos educativos que transmiten los principales valores, actitudes, normas, creencias y sentimientos.

Desde esta perspectiva, Colomo y De Oña (2014) utilizan el análisis de contenido para examinar canciones con el tema de la muerte que pudieran ser empleadas como recurso didáctico con alumnos de los niveles de secundaria y bachillerato. De manera similar, Martínez y Quiroz (2012) desarrollan una parte del programa escolar de ciencias sociales de secundaria empleando el análisis de las letras de canciones con la intención de propiciar en los alumnos un análisis crítico del mundo que los rodea y así aproximarlos a los contenidos de historia y política. En este sentido, también se han empleado canciones infantiles cuyas 


\section{Revista Iberoamericana \\ de las Ciencias Sociales y \\ Humanísticas}

ISSN: 2395 - 7972

letras se enfocan en temas relacionados con contenidos ecológicos en programas de ciencias naturales de educación primaria (Galicia, Ruiz, Sánchez y Pavón, 2005).

Los ejemplos antes descritos son solo una pequeña muestra de que la música se ha empleado como elemento didáctico para diferentes propósitos, en diversas asignaturas y en distintos niveles educativos, aunque vale acotar que en la universidad no se tienen evidencias hasta el momento.

En efecto, la didáctica del nivel superior como disciplina se encuentra en desarrollo y todavía no es tan reconocida socialmente como en otros niveles educativos. Esto se debe a que recientemente se ha desarrollado la construcción de didácticas específicas o propuestas especiales para cada área de conocimiento. De esta manera se procura vincular la teoría y la práctica en la formación universitaria y la posibilidad de proponer experiencias didácticas innovadoras para la enseñanza en el nivel superior (Moreno, 2011), como el análisis de casos, los grupos de estudio y reflexión, entre otras (Del Regno, 2011).

Aunado a lo anterior, se puede afirmar que en el nivel de posgrado tampoco se tienen muchas evidencias al respecto; por ejemplo, en la Maestría en Psicología con Residencia en Terapia Familiar se impulsa a los alumnos a que desarrollen materiales que permitan reflejar el manejo de los conceptos adquiridos en la formación profesional o que puedan emplear en su práctica profesional, ya sea como elementos didácticos para enseñar un determinado tema a estudiantes de pregrado, o bien a poblaciones a las cuales las instituciones de salud les solicitan realizar talleres y conferencias.

Por este motivo, en el presente trabajo se reporta el análisis de letras de canciones para identificar algunos temas presentes en los currículos de la referida maestría; uno de esos temas es el ciclo vital de la familia.

El sistema familiar está en constante cambio. De acuerdo con Minuchin (1974), el desarrollo de la familia transcurre en etapas a lo largo de las cuales sufre variaciones; los períodos de crecimiento pueden provocar transformaciones al sistema y con ello la conformación de nuevas reglas de interacción familiar, tanto en el interior como en el exterior del sistema. Las etapas, que en su conjunto son reconocidas como el ciclo de vida, son las siguientes: formación de la pareja; la pareja con hijos pequeños; la familia con hijos en edad escolar y/o adolescentes, y la familia con hijos adultos. Puede ocurrir que las familias permanezcan atoradas en una etapa aunque requieran de una transformación ante nuevas 


\section{Revista Iberoamericana \\ de las Ciencias Sociales y \\ Humanísticas}

ISSN: 2395 - 7972

situaciones, lo cual las puede conducir a manifestaciones sintomáticas (Montalvo, Espinosa y Pérez, 2013).

Aunque hay diversas propuestas de las etapas del ciclo vital de la familia, este constructo es una herramienta que le da sentido a todo trabajo con familias, sea el objetivo de este la prevención, la intervención (tratamiento o terapia) o la investigación. Retomando las palabras de Estrada (1987), existen dos ventajas al considerar el ciclo vital de la familia: a) ofrece un instrumento de organización y sistematización invaluable para el pensamiento clínico que permite llegar con menos tropiezos al diagnóstico, y b) brinda la oportunidad de revisar casos clínicos dando la pauta para reconocer fenómenos similares en otras familias y que indican, también, las vías que conducen a la intervención terapéutica oportuna.

Ahora bien, en cuanto a los requerimientos exigidos a los alumnos de la Maestría en Terapia Familiar, en una asignatura se les solicitó que determinaran si el ciclo vital de la familia se encontraba incorporado de alguna manera en las letras de las canciones y que analizaran cómo en ellas se proyectaban las diferentes etapas del mencionado ciclo, así como algunas de las principales dificultades que los individuos debían afrontar en el tránsito de una etapa a otra.

Con este análisis, desde una perspectiva didáctica de la asignatura, se pretendía que los alumnos se dieran cuenta de las formas en que los miembros de la familia interactuaban y resolvían sus dificultades, con base en diversos factores que dan contexto a sus interacciones y que son validadas o perpetuadas socialmente a través de las canciones. En este análisis, se consideraron los aspectos sociales y culturales presentes en la época en que fueron compuestas para hacer visibles las premisas y presiones sociales que influyen en la resolución de algunas dificultades en las crisis de transición en las etapas de la vida familiar. Cabe destacar que esta tarea, además de constituir un ejercicio en el que se aplicarían los conocimientos adquiridos, también responde a la creación de material didáctico para los fines descritos en el párrafo anterior.

Explicado esto, se puede afirmar que los objetivos del presente reporte fueron analizar cómo el ciclo vital de la familia se encuentra incorporado en las letras de las canciones y mostrar el empleo de las canciones como recurso didáctico en estudios de nivel superior. 


\section{Revista Iberoamericana \\ de las Ciencias Sociales y \\ Humanísticas}

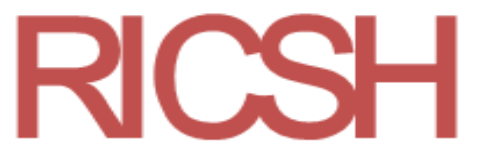

ISSN: $2395-7972$

\section{Marco conceptual}

En este trabajo se recurrió al análisis de contenido, una técnica que posibilita interpretar los productos comunicativos vertidos en los textos, mensajes o discursos con el fin de realizar inferencias reproducibles y válidas (Krippendorff, 2004; Piñuel, 2002). Este análisis, en concreto, se desarrolla dentro de un modelo de comunicación en el que se infiere sobre las experiencias, sentimientos y otros elementos del comunicador. Para ello, se divide el material en unidades interpretables que se pueden triangular con los resultados de otros estudios. Al respecto, Abela (2002) sostiene que este procedimiento permite profundizar en el contenido y en el contexto social donde el mensaje se desarrolla.

Para el análisis del sistema familiar representado en las canciones se tomaron como elementos centrales a) el ciclo vital de la familia, atendiendo la propuesta de Haley (1980), quien considera a la familia como una unidad en constante cambio que recorre un proceso de desarrollo de seis etapas: noviazgo, primeros tiempos de matrimonio, nacimiento de los hijos y trato con ellos, periodo intermedio del matrimonio, destete de los padres respecto a sus hijos y retiro de la vida activa y vejez; asimismo, se consideraron b) las pautas relacionales $y$ de comunicación, entendidas como las interacciones entre los miembros de una familia, cuyo lenguaje tanto analógico como digital hace referencia al tipo de vínculo que se da en el interior del sistema familiar en lo cotidiano. Estas pautas pueden agruparse en aquellas asociadas con la estructura familiar, como las posiciones y los roles, y las que están presentes durante el proceso de conformación familiar (Minuchin, 1974). También se tomaron en cuenta c) las premisas de realidad o premisas de vida, que, de acuerdo con Bateson (1972), son las premisas epistemológicas o creencias que se sustentan en las percepciones básicas de las personas con las que se selecciona y remodela la realidad, de manera que esta resulte congruente con las propias creencias acerca de la clase de mundo en el que se vive. Esto implica que esas ideas están interconectadas con las de aquellas personas involucradas en el propio ecosistema sociocultural. 


\section{Revista Iberoamericana \\ de las Ciencias Sociales y \\ Humanísticas}

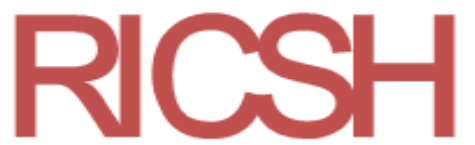

ISSN: $2395-7972$

\section{Método}

Inicialmente, la muestra o corpus de trabajo estuvo integrado por 30 canciones seleccionadas en Internet mediante el buscador de Google. En el recuadro de búsqueda, se ingresaron las palabras familia y español, y se seleccionaron las cuatro primeras páginas referidas por Google, asumiendo que el buscador organiza los resultados de acuerdo con su popularidad en un orden descendente. De las canciones contenidas en cada página se eligieron aquellas que aludieran a relaciones familiares en las que estuvieran presentes o se pudieran identificar tres elementos: a) ciclo vital de la vida familiar, b) pautas relacionales y de comunicación y c) premisas de realidad.

En esta primera selección, solo tres canciones cumplieron con los criterios: Amor y control, Se lleva la libertad con él y El gran varón. Luego se realizó una segunda búsqueda empleando los mismos buscadores y añadiendo tres géneros musicales. La selección condujo a una canción que cumplió con los tres elementos: El gran varón, perteneciente al género musical salsa. Esta fue la canción seleccionada para el presente trabajo.

\section{Procedimiento}

Se realizaron tres análisis: en el primero se expusieron aspectos como el origen, la edad, el sexo y la raza del compositor y del cantante. En el segundo se identificaron elementos fundamentales de la canción, tales como pertinencia del título, coherencia del mensaje y si el hecho era real o ficticio. En el tercero se abordó de lleno el análisis en función del ciclo vital de la familia, especialmente sobre las interacciones familiares inferidas por la descripción realizada en la letra de la canción, haciendo referencia a importantes aspectos de corte sistémico.

\section{Resultados \\ Primer análisis}

- Canción: El gran varón.

- Autor: Omar Alfanno. Nació en Santiago de Veraguas de Panamá. Empezó formalmente su carrera como compositor con el tema El gran varón, el cual es cantado por Willie Colón. 


\section{Revista Iberoamericana \\ de las Ciencias Sociales y \\ Humanísticas}

ISSN: $2395-7972$

- Intérprete: Willie Colón. Conocido maestro del trombón y uno de los representantes de la fusión de distintos ritmos caribeños, afroamericanos y de la música urbana. Es un músico estadounidense, nieto de migrantes puertorriqueños. Nació el 20 de abril de 1950 y creció en el barrio latino del distrito del Bronx, un hecho que ha marcado gran parte de su trayectoria personal y artística, pues implica rebeldía y trasgresión de las normas establecidas.

\section{Segundo análisis}

La canción se titula El gran varón porque hace alusión a las expectativas del modelo de padre tradicional en los años sesenta, época en donde se ubica el relato y donde todavía se esperaba en algunas comunidades que el primogénito fuera un varón que siguiera los pasos de su padre, en términos de la tradición profesional de la familia y las ideas de masculinidad.

El título está relacionado directamente con el contenido de la canción, la cual narra una discriminación que no da lugar a la diversidad sexual, que es castigada con el destierro familiar del miembro "desviado".

La composición hace referencia a un hecho real. El protagonista fue compañero de secundaria/bachillerato de Omar Alfanno, quien le puso por seudónimo Simón. En la vida real, ese compañero se había ido a Los Ángeles, California (Estados Unidos de Norteamérica), para sentirse libre de los prejuicios de la comunidad en que vivía. Allá muere solitariamente en el hospital a causa del sida.

La versión original es interpretada por Willie Colón; refiere que Simón nace en 1956 y muere en 1986. El gran varón está incluida en el disco Top secret (Fania Records, 1989).

\section{Tercer análisis. Análisis sistémico de la canción El gran varón}

En esta sección se presentan fragmentos de la canción en negritas y cursivas, seguidos del análisis correspondiente:

En la sala de un hospital a las 9 y 43 nació Simón es el verano del 56, el orgullo de don Andrés por ser varón.

a) El ciclo vital de la familia: El nacimiento de los hijos es una etapa importante en este ciclo. Haley (1980) menciona que con el nacimiento de un hijo la joven pareja se introduce más en la red total de parientes, en cuanto se modifica la índole de los viejos vínculos y se forman otros nuevos. Aunque en la canción no se hace alusión a ninguno de 


\section{Revista Iberoamericana \\ de las Ciencias Sociales y \\ Humanísticas}

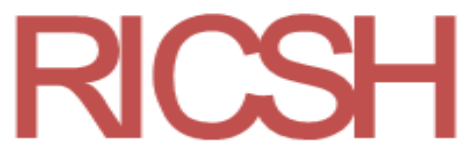

ISSN: 2395 - 7972

estos elementos, hay que considerar que se encuentran presentes en la familia de don Andrés y Simón.

b) Las pautas relacionales y de comunicación: En estos versos no se pueden apreciar las relaciones familiares típicas del patriarcado latino, prevalentes a finales de los 50, pero en estrofas subsiguientes se podrán inferir.

c) Las premisas de realidad o premisas de vida: La premisa presente versa sobre la importancia de ser hombre, la implicación que tiene la masculinidad dentro del seno familiar y del núcleo social. En el enunciado se advierte cómo se refrenda la masculinidad del padre cuando el primogénito es de sexo masculino y por ello generador de orgullo. Esta idea es compartida socialmente en la época que se narra en la canción.

Fue criado como los demás, con mano dura, con severidad, nunca opinó.

a) El ciclo vital de la familia: No se precisa la etapa del ciclo vital, pero se puede inferir que es en la etapa de la familia con hijos pequeños cuando se presentan más claramente los estilos de crianza.

b) Las pautas relacionales y de comunicación: En los años 50 cada uno tenía su lugar: por ejemplo, los padres, maestros y adultos mayores debían ser tratados con respeto por parte de los más jóvenes. En el seno de la familia, la figura del padre era la que daba órdenes e imponía sanciones; además, era incuestionable, hecho reflejado en estas líneas. Cuervo (2010) apunta que los estilos autoritarios y castigadores contribuyen a un desarrollo emocional disfuncional y deficitario en estrategias y competencias emocionales para la adaptación en distintos contextos a lo largo de la vida. Dentro de las pautas de comunicación que caracterizan a la familia con un estilo autoritario está la rigidez y la unidireccionalidad.

c) Las premisas de realidad o premisas de vida: Aquí se refleja la creencia popular de la época de que la disciplina rígida es necesaria para que los hijos aprendan a obedecer y a aceptar sin cuestionar. Esta disciplina, entendida o clasificada como autoritaria, era estimada como la mejor forma de educar a los niños y garantizar el apego a las normas sociales.

De acuerdo con Aunola, Stattin y Nurmi (2000), los padres con un estilo de crianza autoritario desarrollan a sus hijos como personas altas en demandas y bajas en su capacidad de respuesta. Por su parte, Baumrind (2012) explica que los hijos de padres autoritarios se manifiestan más hostiles y con menos control, poca afiliación con sus compañeros, además 


\section{Revista Iberoamericana \\ de las Ciencias Sociales y \\ Humanísticas}

ISSN: 2395 - 7972

de que se muestran más inseguros, aprehensivos y hostiles, de ahí que no opinen, como se refiere en la canción que le aconteció a Simón.

El estilo de crianza autoritario tuvo una aceptación social durante mucho tiempo en décadas pasadas. Cuando en la letra de la canción se menciona que fue criado como los demás, se puede suponer que hace alusión no solo a los posibles hermanos, sino a la comunidad en la que se insertan Simón y su padre. En la época que se narra en la canción, se esperaba que un padre fuese un agente disciplinario extremo para ser considerado socialmente como ejemplar. El estrés que genera en el padre cumplir tal expectativa puede generar un mayor autoritarismo e incluso violencia. Se sabe que los estilos autoritarios pueden ser influidos por circunstancias ambientales generadoras de estrés en los padres (Radziszewska, Richardson, Dent y Flay, 1996). Además de las circunstancias antes mencionadas, es factible que el autoritarismo ejercido por don Andrés sea fortalecido por un factor exosistémico, el cual podría corresponder a la comunidad en la que padre e hijo están inmersos. Se ha mencionado que las comunidades que sufren inseguridad, baja cohesión entre los vecinos y creencias favorables sobre el castigo incrementan la posibilidad de reproducir un método disciplinario violento y de abuso infantil.

Cuando crezcas vas a estudiar la misma vaina que tu papá, óyelo bien, tendrás que ser un gran varón.

a) El ciclo vital de la familia: Es posible inferir que Simón se encuentra en la adolescencia porque debe empezar a considerar el campo de estudio que elegirá para posteriormente desarrollarse profesionalmente. Se ha mencionado que una de las funciones de la familia es la de proveer refugio, protección, afecto, apoyo y asistencia a modelos normativos y a los vertiginosos cambios sociales, así como a la creciente individualidad (Millán de Benavides, 2013). En este caso, vemos que al hijo se le obliga a estudiar lo que la familia y el padre desean para brindarle dicha protección. Por otra parte, hay que señalar que el empleo del vocablo vaina - a pesar de la imprecisión de su significado en el uso popular colombiano - también alude a un elemento de protección, pues se aplica a la envoltura tierna y alargada en la que están encerradas las semillas de algunas plantas. Así pues, puede interpretarse que cuando se hace referencia a que Simón debe estudiar lo mismo que el padre, esto supondría un resguardo a los cambios externos, manteniendo una estructura familiar intacta.

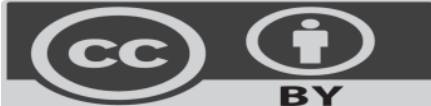




\section{Revista Iberoamericana \\ de las Ciencias Sociales y \\ Humanísticas}

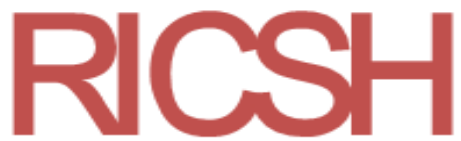

ISSN: $2395-7972$

b) Las pautas relacionales y de comunicación: En estos versos nuevamente se vuelve a representar la parte autoritaria del padre, así como la rigidez y la unidireccionalidad que caracterizan a la familia con un estilo autoritario. No hay opciones: Simón tiene que elegir la misma carrera que don Andrés, el padre, quien seguramente estudió lo mismo que su padre. La frase óyelo bien es imperativa, un mandato. Puntualiza el deber sobre el ser; un deber marcado por la estructura familiar, social y religiosa en cuanto al seguimiento de costumbres e identidades profesionales que impone la orientación sexual del joven.

c) Las premisas de realidad o premisas de vida: Dentro de las premisas de realidad se puede aludir a la arraigada creencia popular de que la orientación sexual se debe únicamente a factores biológicos. Además, según las creencias de la época en que ocurre la situación narrada, la única opción posible es ser un gran varón. Es factible interpretar que estas ideas están enfatizadas por el uso de la palabra vaina, que podría ser entendida también como un elemento figurativo que implicaría la presencia de semillas encerradas en el fruto de una planta. En este caso es el padre quien dota las semillas, es decir, las mismas características biológicas.

Las creencias populares se ven fortalecidas, por ejemplo, por los discursos religiosos. Según Vaggione (2008), la Iglesia mantiene la premisa de defender a la familia como forma de proteger a Latinoamérica, amenazada por los movimientos feministas y de diversidad sexual; por ello, la institucionalización de un modelo único de familia también se sostiene por la influencia de los actores y discursos religiosos en la población, sin olvidar otros factores manejados por el Estado.

Al extranjero se fue Simón, lejos de casa se le olvidó aquel sermón. Cambió la forma de caminar, usaba falda, lápiz labial y un carterón.

a) El ciclo vital de la familia: En este fragmento es posible observar la etapa del ciclo de vida familiar en el cual los hijos se van del hogar parental para construir una vida independiente con base en su propia identidad; esta es la etapa de la emancipación o destete de los padres, según Haley (1980). Las interacciones entre padres e hijos dan un giro importante, ya que la jerarquía parental antes intocable es ahora cuestionada a través de los comportamientos de los hijos. En dicho periodo es fundamental que los padres logren mantenerse al margen de las decisiones de sus hijos, al mismo tiempo que sostienen un vínculo de apoyo y guía. A su vez, los hijos estarán en posición de tomar decisiones propias 


\section{Revista Iberoamericana \\ de las Ciencias Sociales y \\ Humanísticas}

ISSN: $2395-7972$

sin buscar la aprobación de sus padres, asumiendo las responsabilidades que ello implica, al tiempo que la relación con sus padres se mueve hacia la horizontalidad (Haley, 1980).

En esta parte de la canción se advierte cómo Simón ha buscado su propia autonomía e identidad. También ha tomado decisiones trascendentales sin considerar la aprobación de su padre, incluso las relativas a su orientación sexual, pues se le olvidó aquel sermón. En la toma de decisiones de Simón, la canción no alude a los padres ni a don Andrés, por lo que se supone que este logra mantenerse al margen.

b) Las pautas relacionales y de comunicación: De acuerdo con Minuchin y Fishman (2004), las reglas y pautas relacionales que rigen a algunas familias tradicionales (como la de Simón) son rígidas al exterior y con límites difusos al interior, lo cual da lugar a un estilo de interacción fusionado, en donde el espacio vital para cada miembro de la familia es insuficiente para el desarrollo de la individualización de sus miembros. Esto es referido en los enunciados de la canción que anteceden los de esta sección. Tal individualización insuficiente de los integrantes puede conducir a que no exista una verdadera compenetración, conocimiento o confianza entre ellos. Por lo anterior, es probable que la familia de Simón no advirtiera manifestaciones que dieran pauta a pensar en que él podría asumir una identidad de género diferente a la que biológicamente le correspondería y se esperaría socialmente.

c) Las premisas de realidad o premisas de vida: Las premisas asumidas en el hogar primario se transformaron radicalmente al cambiar de contexto, pues en la letra se refiere que lejos de casa olvida la censura familiar. La manera de concebir la realidad es otra, propiciada por las premisas que caracterizan el contexto social en el cual se ha insertado.

La identidad implica aprender a mirarse los unos a los otros a partir de los patrones culturales, a través de los cuales se distinguen a aquellos que no son formados dentro de ese contexto cultural. En ese sentido, para Bartra (2016) la pertenencia a una cultura es la carta de identidad que se presenta a la mirada del otro. Al cambiar de contexto cultural, al ir al extranjero, Simón se aparta de los cánones sociales originales y empieza a adoptar otros, en especial los vinculados con su preferencia sexual, la cual se fortalece incluso propiciando que Simón buscara el cambio de apariencia: usaba falda, lápiz labial y un carterón. Lo anterior sugiere que Simón está siguiendo los patrones culturales del contexto social en el que se encuentra, configurando de esta manera una realidad nueva acorde con su identidad de género. 


\section{Revista Iberoamericana de las Ciencias Sociales y Humanísticas}

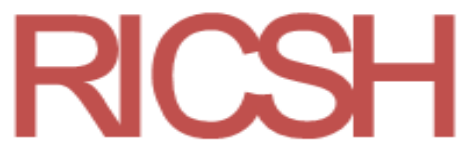

ISSN: $2395-7972$

Solórzano y Mendoza (2014) comentan que las personas homosexuales han sido socializadas inicialmente en contextos donde se espera la heterosexualidad de sus miembros. Por lo tanto, la construcción de su identidad requiere de un largo proceso a través del cual la persona va adquiriendo conciencia mediante la negociación y consolidación de su orientación sexual en otros contextos. A diferencia de otros grupos minoritarios, no tienen la posibilidad dentro de su grupo primario de aprender y expresar con libertad su identidad de género. El contexto social en el que Simón nació estaba determinado por reglas y pautas rígidas relativas a los roles de género. Pero al cambiar el contexto del hogar de origen, las reglas y pautas se flexibilizan. Estas ahora son determinadas en función de lo socialmente permitido en el país al que se mudó. Esto, aunado a que Simón está en la etapa de autonomía y confirmación de la identidad, podrían ser los factores que explicarían el hecho de que él asumiera plenamente su orientación sexual de manera abierta.

Cuenta la gente que un día el papá fue a visitarlo sin avisar, vaya qué error. Una mujer le habló al pasar, le dijo: “Hola, ¿qué tal, papá? ¿Cómo te va?”. “ ¿No me conoces? Yo soy Simón; Simón tu hijo, el gran varón”.

a) El ciclo vital de la familia: La narración continúa en la etapa en la cual los hijos se van del hogar parental. Como se mencionó, los padres todavía establecen un vínculo de apoyo o guía, por lo que don Andrés va en búsqueda de su hijo, ya fuese para darle apoyo o para ver si continuaba con los preceptos por él establecidos. Pero no tiene en cuenta que en esta etapa ya no tiene la misma jerarquía y que se deben establecer relaciones tendientes a la horizontalidad, de ahí que visitar al hijo sin tomar en cuenta su aprobación implicaría imponer su jerarquía de padre y no establecer un vínculo más horizontal. Este error se pone de manifiesto en la canción.

b) Las pautas relacionales y de comunicación: Watzlawick (1971) señala que en una situación comunicativa, toda conducta, actividad, inactividad, silencio o inatención tiene un valor como mensaje e influye en el comportamiento de los demás, quienes, a su vez, emiten una respuesta. En este sentido, la estrofa aquí referida da oportunidad de apreciar cómo, de alguna forma, Simón había comunicado al padre su orientación sexual, recibiendo como respuesta la omisión, es decir, un silencio que comunicaba. Esto podría implicar no solo el asombro del padre, sino también el rechazo a la nueva realidad que su hijo le presentaba, de ahí que hubiese una réplica de Simón que se hizo necesaria en ese momento: ¿No me conoces? Yo soy Simón; Simón tu hijo, el gran varón. 


\section{Revista Iberoamericana \\ de las Ciencias Sociales y \\ Humanísticas}

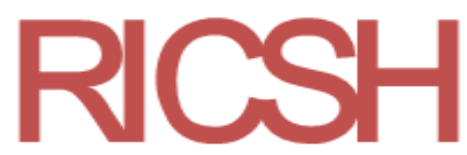

ISSN: $2395-7972$

c) Las premisas de realidad o premisas de vida: En esta parte de la canción se aprecia el momento en el que la realidad del hijo converge con la del padre, pero chocan. Cuando don Andrés, el padre de Simón, llega sin previo aviso se encuentra frente a la nueva realidad construida por su hijo, quien ha dejado atrás todos los preceptos autoritarios y de género profesados en su familia y en su comunidad anterior para adaptarse al nuevo contexto cultural en el que vive actualmente. Ha pasado exitosamente por el proceso de construcción de una nueva identidad sexual, e incluso encuentra el momento oportuno para expresarse abiertamente ante su padre.

El silencio de don Andrés, que se sugiere en la letra de la canción, se puede interpretar como la incomprensión del padre ante las nuevas premisas de vida de su hijo: emancipación y abierto desafío a la autoridad cultural, familiar y paternal, en un grado tal que lo expresa libremente. La noción del padre como autoridad familiar y la orientación sexual profesada desde las ideas del patriarcado quedan vulnerables ante la actitud que Simón presenta a su padre.

No se puede corregir a la naturaleza, palo que nace doblado, jamás su tronco endereza.

No se puede corregir...

Se dejó llevar por lo que dice la gente, su padre jamás le habló, lo abandonó para siempre.

No se puede corregir...

No te quejes Andrés, no te quejes por nada. Si del cielo te caen limones aprende a hacer

limonadas.

a) El ciclo vital de la familia: La narración de la vida de Simón descrita en este fragmento se encuentra ubicada plenamente en la etapa en la cual los hijos se van del hogar parental. Como se señaló, aunque los hijos tienen autonomía, se sugiere que los padres establezcan con ellos una relación más horizontal, sin dejar de representar un vínculo de apoyo o guía. En este caso, don Andrés no cumple con tal vínculo, de ahí que se le recrimine: Su padre jamás le habló, lo abandonó para siempre.

b) Las pautas relacionales y de comunicación: Parece ser que Simón y don Andrés, especialmente este último, entienden la autonomía como dejar de entablar conversaciones (su padre jamás le habló). Sin embargo, esta actitud que en apariencia pudiera interpretarse como una falta de comunicación, pero en realidad es una evidencia de que para las personas aludidas en la canción la autonomía implica el separarse totalmente rompiendo vínculos entre ellos. Esto conduce, por una parte, a que Simón cambie y, por otra, a que don Andrés se 


\section{Revista Iberoamericana \\ de las Ciencias Sociales y \\ Humanísticas}

ISSN: 2395 - 7972

desentienda completamente de Simón, en la suposición de que no seguirá los preceptos inculcados en su familia.

c) Las premisas de realidad o premisas de vida: El párrafo citado previamente centra su discurso en la imposibilidad de modificar a la naturaleza (No se puede corregir a la naturaleza), pues se tiene como premisa que se nace de una forma característica sin posibilidad de alterarla. Las posturas teóricas describen que la identidad de género es una construcción social y cultural; no obstante, en la letra de la canción se alude a la conseja popular y la aplica en el caso de Simón sugiriendo que su orientación sexual se debe a factores biológicos, quizás equivocados, porque se menciona que palo que nace doblado...; además, se afirma que no se puede hacer nada al respecto: jamás su tronco endereza.

Asumir la realidad es la conseja: No te quejes Andrés, no te quejes por nada, pero se presenta como castigo, ya sea porque don Andrés no cumple con la horizontalidad en las relaciones padre-hijo esperadas para la etapa del ciclo vital, o bien porque la comunidad, en esa época, ve los cambios de identidad sexual de género como algún error biológico como resultado de relaciones sociales inadecuadas (Se dejó llevar por lo que dice la gente) o como un castigo divino ante los cuales no se pude hacer nada. El que don Andrés se desentienda de Simón se produce porque no ve estas cuestiones y presupone que como varón seguirá los pasos de su padre.

$Y$ mientras pasan los años, el viejo cediendo un poco.

Simón ya ni le escribía Andrés estaba furioso.

Por fin tuvo noticias de donde su hijo estaba. Andrés nunca olvidó el día de esa triste llamada.

a) El ciclo vital de la familia: Andrés se encuentra en la etapa del ciclo vital denominada sufrimiento de la vejez; en esta una persona mayor puede encontrar una función útil, aunque otras veces, en la medida en que los tiempos cambian, los viejos son vistos como carentes de importancia para la acción de la generación más joven. Por la letra de la canción parece que esta última situación es en la que se encuentra don Andrés.

De acuerdo con Rodríguez (1998), la vejez se construye en oposición a la juventud en lo que respecta a la movilidad, salud, pasión y juicio; pero en lo relativo a conocimiento de la vida, a esta etapa se le atribuye la sabiduría. Dentro de los refranes, que suelen ser buenos indicadores de la mentalidad tradicional, encontramos ideas tales como "Donde no 


\section{Revista Iberoamericana \\ de las Ciencias Sociales y \\ Humanísticas}

ISSN: 2395 - 7972

hay viejo no hay buen consejo" y "Dentro del consejo, la lengua del viejo". Pero este no es el caso de don Andrés.

b) Las pautas relacionales y de comunicación. Simón evita hablar con su padre, pero eso también es una forma de comunicarse y relacionarse, y puede ser producto del desarrollo emocional disfuncional carente de estrategias y competencias emocionales debido al estilo autoritario al que estuvo expuesto. Las familias autoritarias representan un factor en la persona, relacionado a la inseguridad, evitación de los problemas, afrontamientos desadaptativos, depresión y soledad asociada al rechazo y desinterés de los padres (Richard de Minzi, 2005).

c) Las premisas de realidad o premisas de vida: En apariencia, se alude a un recuerdo aleccionador. Koestler (citado por Watzlawick, 1971) describe las relaciones familiares y la pertenencia a un plano donde no rigen las normas corrientes de juicio y la conducta en un laberinto de tensiones, disputas y reconciliaciones, cuya lógica es autocontradictoria. Se trata de un universo saturado de recuerdos de los que no se aprende nada, saturado de un pasado que no proporciona orientación para el futuro. En este universo, después de cada crisis y cada reconciliación, el tiempo comienza de nuevo y la historia siempre está en el año cero.

De acuerdo con lo citado por Koestler, la relación que tiene don Andrés con Simón es un pasado del cual no se aprende nada, ya que el intento por no comunicar comunicaba la molestia del padre hacia el hijo; es una crisis, pero sin reconciliación de uno mismo consigo mismo, valores y criterios distorsionados por el contexto social y cultural de una lógica autocontradictoria.

De una extraña enfermedad murió Simón. Es el verano del 86.

Al enfermo de la cama 10 nadie lloró.

Hay que tener compasión, basta ya de moraleja. El que esté libre de pecado que tire la primera piedra.

El que nunca perdona tiene el destino cierto

de vivir amargos recuerdos en su propio infierno.

a) El ciclo vital de la familia: De alguna manera, en esta historia no se refleja el ciclo vital de la familia, pues entre sus diversas clasificaciones se presentan como últimas etapas la vejez y la muerte de los padres; no obstante, en este caso el que muere es el hijo, Simón. 


\section{Revista Iberoamericana \\ de las Ciencias Sociales y \\ Humanísticas}

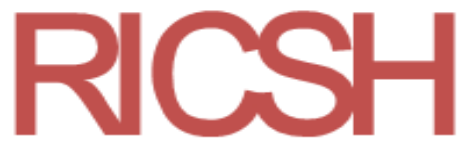

ISSN: 2395 - 7972

La muerte de un hijo rompe las expectativas de los padres y de toda la familia; Haley (1980) señala que cuando la transición de una etapa del ciclo vital a otra se disloca aparecen diversos síntomas que aquejan a toda la familia. En la canción no se reflejan estos síntomas, pero es de esperarse que ocurran, principalmente porque la situación narrada no cumple con la expectativa de que los padres mueran primero. Haley menciona que estos síntomas se expresan en función de la situación social, que también cambia a través del tiempo. De alguna manera, esto representa otra importante razón para indagar en las nuevas formas de organización familiar, ya que Haley consideraba como cambiante la conceptualización de familia y por lo mismo debiéramos actualizar nuestra comprensión de la misma.

b) Las pautas relacionales y de comunicación: Es muy probable que en la mentalidad tradicional de don Andrés prevaleciera su papel jerárquico como padre y jefe de familia. Lo anterior le hace suponer que Simón era quien debería buscar una reconciliación y pedir perdón, ya que había incurrido en el rompimiento de las normas morales y sociales inculcadas desde el seno familiar. En ese contexto, un acercamiento del padre hacia el hijo sería interpretado como un signo de debilidad por parte de don Andrés; además, sería la evidencia, entre otras cosas, de que fue el padre (máxima autoridad familiar y poseedor de la verdad absoluta) quien se había equivocado. De forma adicional, y retomando lo referido por Richard de Minzi (2005), es posible que Simón tuviera la tendencia a optar por la evitación de los problemas, tal como ocurre con algunas personas que provienen de familias con un estilo de crianza autoritario, y por ello no hubiese aprendido o desarrollado una variedad de estilos de afrontamiento congruentes con las situaciones que se le presentaran. Lo anteriormente expuesto podría explicar cómo es que se perpetró la ruptura de la comunicación entre don Andrés y Simón.

c) Las premisas de realidad o premisas: Con la conseja popular el que esté libre de pecado que tire la primera piedra, en la canción se deja libre de culpa a don Andrés y a Simón, de modo que la solución de los conflictos se remite al perdón para no sufrir. Pedir y recibir perdón tiene un sentido bidireccional. Para Ricoeur (2004) los efectos de una falta (daño o herida) y de su posible perdón cruzan todas las operaciones constitutivas de la memoria, la historia de los individuos y marcan el olvido de lo sucedido de un modo particular. El perdón siempre está al servicio de una finalidad (rescate, redención, salvación, reconciliación) o intenta establecer una "normalidad" (psicológica o moral). Estas manifestaciones pueden ser explícitas o implícitas: las primeras de ellas tienen una 


\section{Revista Iberoamericana \\ de las Ciencias Sociales y \\ Humanísticas}

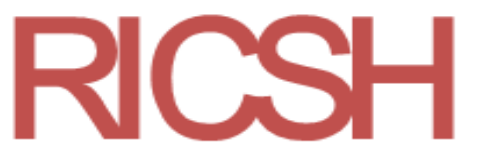

ISSN: $2395-7972$

interacción directa con la persona afectada, mientras que las segundas quedan mediadas de manera indirecta por manifestaciones verbales.

\section{Discusión}

Después de analizar la letra de la canción El gran varón, lo cual se concretó siguiendo una perspectiva sistémica como producto de una actividad didáctica, y mostrado el producto de esta, se propone que el análisis de las canciones podría resultar una vía de acceso a contenidos curriculares del nivel educativo superior. Las canciones, en este ciclo educativo, resultan un medio de expresión poco aprovechado en la enseñanza, en especial de las disciplinas relativas a las interrelaciones personales, como es en este caso la Maestría en Psicología con Residencia en Terapia Familiar.

La experiencia referida y el producto presentado en este trabajo ponen de manifiesto que el análisis de las canciones es una estrategia didáctica que ofrece la posibilidad de promover la reflexión sobre los temas relativos a la formación de los maestrantes. En concreto, con la canción elegida en este trabajo y presentada como ejemplo, los temas que pudieron reflexionarse y debatirse en clase fueron el desarrollo de las fases del ciclo vital de la familia, el modelo heterosexual normativo, la identidad de género y las creencias que sostienen estilos de crianza en una comunidad y en una época determinada.

Debido a la manera en que fue concretada la actividad, los maestrantes refirieron que pusieron en práctica los conocimientos adquiridos de una manera no tradicional y que el ejercicio lo vivenciaron como si fuese una actividad lúdica, lo que trajo como resultado la apropiación del conocimiento de una manera agradable. También comentaron que la experiencia les permitió mirar, o más bien escuchar, de otra manera las canciones y volverse sujetos críticos de los textos que cantan. De hecho, es importante destacar que los alumnos se sintieron reconocidos en lo que respecta a sus gustos musicales.

Dado que la música y las canciones en particular conforman un área muy importante de la esfera cultural de los individuos, el trabajar con estos elementos en un ámbito académico, aparentemente ajeno, les permitió expresar sus aficiones y conocimientos especializados en esa área. De esta manera mostraron aportes a la clase de una manera no tan habitual, pero sí significativa. 


\section{Revista Iberoamericana \\ de las Ciencias Sociales y \\ Humanísticas}

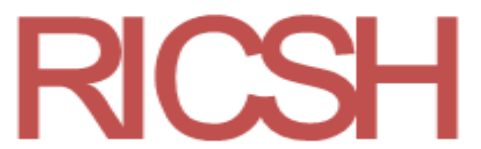

ISSN: 2395 - 7972

Para que las canciones propicien un aprendizaje significativo se requiere de una interpretación. En este sentido, el docente debe formar parte importante del proceso como orientador de la mirada crítica hacia los aspectos que deban ser atendidos de acuerdo con la asignatura que deba ser aprendida y después dejar en libertad a los alumnos para que analicen e incluso incluyan otros aspectos que les pudieran resultar interesantes.

Ya que los textos de las canciones son producto de una carga subjetiva en sí mismos, y por tener de igual forma una intencionalidad al momento de transmitir un mensaje (Martínez y Quiroz, 2012), este debe ser orientado tomando en cuenta las condiciones reales del contexto donde surgieron. En otras palabras, el docente debe promover la reflexión en torno a lo escrito por el autor tomando en cuenta los aspectos históricos e ideológicos presentes en la época en que fue escrita la canción, así como las interpretaciones culturales que se le dan al género musical elegido. De este modo el texto puede ser redimensionado desde el campo de la comprensión para trascender la simple expresión literal.

Por otra parte, vale acotar que este tipo de estrategias posibilitó que los maestrantes se enfocaran no solo en las canciones como una construcción cultural permanente de la sociedad, sino también en otras manifestaciones artísticas en las cuales se podrían examinar los sistemas familiares.

Los textos de las canciones dan cabida a la expresión de las interacciones familiares que surgen en torno a un contexto en constante transformación, tal es el caso de la pieza musical analizada y presentada en esta ocasión. De acuerdo con la mirada sistémica, frente a los cambios las familias logran configurar las interrelaciones necesarias para mantener al sistema unido y en constante funcionamiento. Para algunas familias asumir que uno de sus miembros se aleja del modelo heterosexual normativo resulta todo un cambio que implica, entre otros muchos aspectos, que el periodo del ciclo de la vida en que ocurre sea experimentado de manera muy particular, en este caso por los miembros de la familia aludida en la canción. En ella se infiere, por ejemplo, que existe la pérdida de las expectativas de continuar con la descendencia.

Con respecto a lo narrado en la canción, la comunidad conoce lo sucedido a la familia de don Andrés, reflejo de otras familias que enfrentan situaciones semejantes. Tal conocimiento puede llevar a la comunidad a construir nuevos patrones de crianza, formas de organización familiar, etc. Dicho en otras palabras, la narrativa de esta canción da cuenta de un momento importante relacionado con los cambios ideológicos en torno a las cuestiones 


\section{Revista Iberoamericana \\ de las Ciencias Sociales y \\ Humanísticas}

ISSN: $2395-7972$

de género, salud y derechos humanos. Asimismo, refleja la necesidad de estructurar una nueva forma de familia, en cuya dinámica se destierren prácticas discriminatorias y se desarrollen interacciones que posibiliten incluir a todos sus integrantes con independencia de su orientación y/o preferencia sexual.

\section{Conclusiones}

Desde una perspectiva pedagógica, el caso particular de esta experiencia representó un caso en el que se aplicaron algunos principios de la pedagogía crítica al incorporar a las canciones como elementos de las construcciones culturales como parte fundamental en el proceso formativo del educando. También fue considerado el aspecto social, al tomar en cuenta los valores familiares y sociales de la época histórica en que fue escrita la narrativa de la canción. Esto permitió que los estudiantes visualizaran los productos culturales de una forma crítica.

Por otra parte, los conocimientos impartidos en la asignatura de carácter psicológico no se aplicaron mecánicamente, pues la situación generada posibilitó que se desarrollaran algunas de las habilidades cognitivas, tales como la conceptualización, la resolución de problemas, la interpretación, la construcción de opiniones, la abstracción, la inferencia, la generalización y el análisis.

Si tuviera que clasificarse esta experiencia como una estrategia de enseñanza, de acuerdo con su momento de aplicación, podría decirse que es posintruccional, pues el análisis de la canción fue realizado después de haber visto el contenido en clase. Es decir, luego de haber revisado el tema El ciclo vital de la familia en la asignatura por medio de exposiciones de los alumnos, se les pidió que buscaran una canción donde pudieran aplicar los conocimientos adquiridos. Por lo general, las estrategias posinstruccionales permiten al alumno valorar su aprendizaje, formar una visión sintética, integradora e incluso crítica del material revisado.

Por rende, se puede afirmar que la canción representa un elemento útil en la educación superior para que los estudiantes ejerciten de una manera no tradicional los contenidos aprendidos en las aulas universitarias. Esta actividad promueve en los alumnos las habilidades de análisis requeridas por la asignatura y es vivenciada como una labor agradable, práctica y asociada a sus afinidades musicales, las cuales fueron resignificadas por ellos desde otro marco más allá del musical y estético.

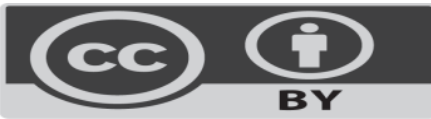




\section{Revista Iberoamericana \\ de las Ciencias Sociales y \\ Humanísticas}

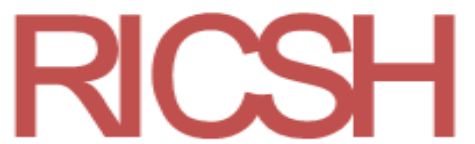

ISSN: $2395-7972$

\section{Referencias}

Abela, A. (2002). Las técnicas de análisis de contenido, una versión actualizada. Centro de Estudios Andaluces, $\quad 10(2), \quad 1-34 . \quad$ Recuperado de http://public.centrodeestudiosandaluces.es/pdfs/S200103.pdf.

Aunola, K., Stattin, H. and Nurmi, J. E. (2000). Parenting styles and adolescents' achievement strategies. Journal of Adolescence, 23(2), 205-222.

Bartra, R. (2016). La jaula de la melancolía. Identidad y metamorfosis del mexicano. México: Debolsillo

Bateson, G. (1972). Pasos hacia una ecología de la mente. Buenos Aires: Lohlé-Lumen.

Baumrind, D. (2012). Differentiating between Confrontive and Coercive Kinds of Parental Power-Assertive Disciplinary Practices. Human Development, 55(2), 35-51. Doi: $10.1159 / 000337962$

Berrocal, E. y Gutiérrez, J. (2002). Música y género: análisis de una muestra de canciones populares. Comunicar: Revista Científica Iberoamericana de Comunicación y Educación, (18), 187-190.

Colomo, E. (2014). Las canciones como recurso didáctico para educar en el amor. Eufonía. Didáctica de la Música, (61), 76-81.

Colomo, E. y De Oña J. (2014). Pedagogía de la muerte. Las canciones como recurso didáctico. REICE. Revista Iberoamericana sobre Calidad, Eficacia y Cambio en Educación, 12(3), 109-121.

Cuervo, A. (2010). Pautas de crianza y desarrollo socioafectivo en la infancia. Diversitas: Perspectivas en Psicología, 6(1), 111-121.

Cuesta, O. y Gómez, A. (2013). La presencia del pensamiento narrativo en la letra de las canciones. Mercadeo, 9(17), 153-170.

De la Peza, M. (2001). Bolero y la educación sentimental en México. México: Universidad Autónoma Metropolitana. Unidad Xochimilco (UAM).

Del Regno, P. (2011). Estrategias de enseñanza del profesor en el aula de nivel superior. Desafíos para la didáctica y la formación docente de dicho nivel. Anuario de Investigaciones en Ciencias de la Educación, 288-303. Recuperado de http://www.filo.uba.ar/contenidos/investigacion/institutos/Iice/ANUARIO_2011/tex tos/19.Del\%20_Regno.pdf.

\section{(c)}




\section{Revista Iberoamericana \\ de las Ciencias Sociales y \\ Humanísticas}

ISSN: $2395-7972$

Estrada, L. (1987). El ciclo vital de la familia. México: Posadas.

Galicia, I. X., Ruiz, C., Sánchez, A. y Pavón, F. (2005). Incidencia de las canciones ambientales en la apropiación de contenidos y valores ecológicos. Revista Intercontinental de Psicología y Educación, 7(1), 21-30.

Gallucci, M. (2008). Análisis de la imagen de la mujer en el discurso de reggaetón. Opción, 55, 84-100.

Haley, J. (1980). Terapia no convencional: las técnicas psiquiátricas de Milton Erickson. Buenos Aires: Amorrortu.

Hormigos, J. (2010). Distribución musical en la sociedad de consumo. La creación de identidades culturales a través del sonido. Dossier Comunicar, 34(12), 91-98.

Iglesias, A. M. (2011). La canción francesa como transmisora de ideología: análisis lingüístico de la canción "La bête” de Zebda. Synergies Espagne, (4), 77-84.

Juliano, D. (1998). Las que saben. Subculturas de mujeres. Madrid: Horas y Horas.

Krippendorff, K. (2004). Content analysis: An introduction to its methodology. London: SAGE.

Martínez, I. A., y Quiroz, R. E. (2012). ¿Otra manera de enseñar las ciencias sociales? Tiempo de Educar, 13(25), 85-109.

Millán de Benavides, C. (2013). Estudios de familia y agendas emergentes. Revista VIA IURIS, (15), 105-111.

Minuchin, S. (1974). Familias y terapia familiar. México: Gedisa.

Minuchin, S. y Fishman, C. (2004). Técnicas de terapia familiar. Buenos Aires: Paidós.

Montalvo, J., Espinosa, M. R. y Pérez, A. (2013). Análisis del ciclo vital de la estructura familiar y sus principales problemas en algunas familias mexicanas. Alternativas en Psicología, 17(28), 73-91.

Moreno, T. (2011). Didáctica de la educación superior: nuevos desafíos en el siglo XXI. Revista Perspectiva Educacional, 50(2), 26-54.

Muñoz-Hidalgo, M. (2007). Bolero y modernismo: la canción como literatura popular. Literatura $y \quad$ Lingüística, (18), 101-120. Recuperado de http://www.scielo.cl/scielo.php?script=sci_arttext\&pid=S0716-58112007000100005

Piñuel, J. (2002). Epistemología, metodología y técnicas de análisis de contenido. Estudios de Sociolingüística, 3(1), 1-42. 


\section{Revista Iberoamericana \\ de las Ciencias Sociales y \\ Humanísticas}

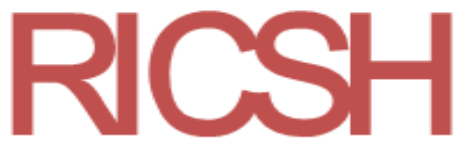

ISSN: $2395-7972$

Radziszewska, B., Richardson, J., Dent, C. and Flay, B. (1996). Parenting Style and Adolescent Depressive Symptoms, Smoking, and Academic Achievement: Ethnic, Gender, and SES Differences. Journal of Behavioral Medicine, 19(3), 289-305. Doi: doi.org/10.1007/BF01857770

Richard de Minzi, M. (2005). Estilos parentales y estrategias de afrontamiento en niños. Revista Latinoamericana de Psicología, 37(1), 47-58.

Ricoeur, P. (2004). La memoria, la historia, el olvido. Buenos Aires: Fondo de Cultura Económica.

Rodríguez, R. F. (1988). La vejez y la muerte. Anales de Psicología, 14(1), 127-135.

Solórzano, A. y Mendoza, M. (2014). "Salir del clóset" en la ciudad de México. Salud Mental, 37(5), 391-397.

Vaggione, J. M. (2008). Las familias más allá de la heteronormatividad. En Motta, C. y Saez, M. (eds.), La mirada de los jueces: género y sexualidad en la jurisprudencia latinoamericana (pp. 13-87). Bogotá: Siglo del Hombre. Recuperado de https://s3.amazonaws.com/academia.edu.documents/33177729/Heteronormatividad. pdf?AWSAccessKeyId=AKIAIWOWYYGZ2Y53UL3A\&Expires=1557655107\&S ignature=8DXvtV5\%2BUxJ6U7PUvOb\%2FaI\%2FIQJ0\%3D\&response-contentdisposition=inline\%3B\%20filename\%3DHeteronormatividad.pdf.

Watzlawick. P. (1971). Teoría de la comunicación humana. Interacciones, patologías y paradojas. Buenos Aires: Tiempo Contemporáneo. 


\section{Revista Iberoamericana de las Ciencias Sociales y Humanísticas}

ISSN: $2395-7972$

\begin{tabular}{|c|c|}
\hline Rol de Contribución & Autor (es) \\
\hline Conceptualización & GALICIA MOYEDA IRIS XOCHITL principal \\
\hline Metodología & $\begin{array}{l}\text { GALICIA MOYEDA IRIS XOCHITL, principal, TAPIA } \\
\text { SANPABLO EVA YURIRIA, apoyo VíQUEZ RODRIGUEZ } \\
\text { JORGE, apoyo }\end{array}$ \\
\hline Software & NO APLICA \\
\hline Validación & $\begin{array}{l}\text { GALICIA MOYEDA IRIS XOCHITL, principal, TAPIA } \\
\text { SANPABLO EVA YURIRIA, colaboración y VíQUEZ } \\
\text { RODRIGUEZ JORGE, colaboración }\end{array}$ \\
\hline Análisis Formal & $\begin{array}{l}\text { VíQUEZ RODRIGUEZ JORGE, principal, TAPIA } \\
\text { SANPABLO EVA YURIRIA, colaborador y GALICIA } \\
\text { MOYEDA IRIS XOCHITL, colaborador }\end{array}$ \\
\hline Investigación & $\begin{array}{l}\text { TAPIA SANPABLO EVA YURIRIA, principal, VíQUEZ } \\
\text { RODRIGUEZ JORGE, colaborador y GALICIA MOYEDA } \\
\text { IRIS XOCHITL, colaborador }\end{array}$ \\
\hline Recursos & $\begin{array}{l}\text { TAPIA SANPABLO EVA YURIRIA, principal, VíQUEZ } \\
\text { RODRIGUEZ JORGE, colaborador y GALICIA MOYEDA } \\
\text { IRIS XOCHITL, colaborador }\end{array}$ \\
\hline Curación de datos & $\begin{array}{l}\text { VíUEZ RODRIGUEZ JORGE,principal, TAPIA } \\
\text { SANPABLO EVA YURIRIA, colaborador Y GALICIA } \\
\text { MOYEDA IRIS XOCHITL colaborador }\end{array}$ \\
\hline $\begin{array}{l}\text { Escritura - Preparación del } \\
\text { borrador original }\end{array}$ & $\begin{array}{l}\text { TAPIA SANPABLO EVA YURIRIA, principal, VíQUEZ } \\
\text { RODRIGUEZ JORGE, colaborador y GALICIA MOYEDA } \\
\text { IRIS XOCHITL, colaborador }\end{array}$ \\
\hline $\begin{array}{l}\text { Escritura - Revisión y } \\
\text { edición }\end{array}$ & $\begin{array}{l}\text { GALICIA MOYEDA IRIS XOCHITL, principal, TAPIA } \\
\text { SANPABLO EVA YURIRIA, colaborador Y VíQUEZ } \\
\text { RODRIGUEZ JORGE, colaborador }\end{array}$ \\
\hline Visualización & $\begin{array}{l}\text { TAPIA SANPABLO EVA YURIRIA, principal, VíQUEZ } \\
\text { RODRIGUEZ JORGE, colaborador y GALICIA MOYEDA } \\
\text { IRIS XOCHITL, colaborador }\end{array}$ \\
\hline Supervisión & GALICIA MOYEDA IRIS XOCHITL, principal. \\
\hline
\end{tabular}


Revista Iberoamericana

de las Ciencias Sociales y

Humanísticas

ISSN: $2395-7972$

Administración de Proyectos $\quad$ GALICIA MOYEDA IRIS XOCHITL . principal

Adquisición de fondos

No aplica.

Vol. 9, Núm. 17 Enero - Junio 2020 